\title{
Multivariate Probabilistic Synthesis of Cellular Networks Teletraffic Blocking with Poissonian Distribution Arrival Rates
}

\author{
Vincent Omollo Nyangaresi ${ }^{1}$, Dr. Silvance Abeka ${ }^{2}$, Prof. Anthony Rodrigues ${ }^{3}$ \\ School of Informatics and Innovative Systems, Jaramogi Oginga Odinga University of Science \& Technology, \\ Kisumu - Kenya.
}

Received: 23 April 2018; Accepted: 25 May 2018; Published: 08 July 2018

\begin{abstract}
Cellular networks are characterized by mobility in which subscribers move freely within the coverage area. Since the radio spectrum is a scarce resource, the available bandwidth is divided by using a combination of Time- and Frequency-Division Multiple Access (TDMA) Code Division Multiple Access (CDMA) and Frequency Division Multiple Access (FDMA). For communication process to succeed, the subscriber must be allocated some frequency band (FDMA), a time slot (TDMA) or pseudorandom binary sequence that modulates the carrier (CDMA). With the increasing number of users, these resources may become unavailable, leading to new call blocking or handover call blocking. Erlang B and Erlang $\mathrm{C}$ have been used in the past to model teletraffic blocking in Public Switched Telephone Network (PSTN). Unfortunately, Erlang B is only ideal when subscribers do not perform call re-attempts after their initial calls are blocked. On the other hand, Erlang $\mathrm{C}$ model is applicable only in networks where queuing is applied and can easily lead to higher blocking rates when the number of users is high. This is because it takes into consideration the number of instances in the queue as well as the resources under use. In this paper, teletraffic blocking probabilities that take into account additional cellular network concepts such as the number of mobile stations, call retries, channels reservation, overlays and underlays, user velocity, relative mobility, call priority, call arrival rates and signal to interference plus noise ratio (SINR) were synthesized. The simulation results showed that the developed teletraffic blocking probabilities were superior to the conventional Erlang B and Erlang C as they consider new concepts that exist in cellular networks that were not envisioned in traditional PSTN.
\end{abstract}

Index Terms: Blocking probability, grade of service, quality of service, teletraffic.

(C) 2018 Published by MECS Publisher. Selection and/or peer review under responsibility of the Research Association of Modern Education and Computer Science

\footnotetext{
* Corresponding author.
}

E-mail address: 


\section{Introduction}

In GSM, a network consists of a number of radio cells in which each cell is assigned a band of frequencies for sending and receiving data. At the center of each cell is base transceiver station (BTS) which is made up of a transmitter, receiver and control unit. To avoid interference, neighboring cells are allocated different frequencies [1]. Unfortunately, as the number of subscribers within a base station increase, the traffic they generate soars up to a point where there are no free time slots or frequency channels to be assigned to new calls. This leads to blocking of either new calls or handed over calls.

According to [2], to offer good Quality of service (QoS), GSM network operators strive to effectively utilize their available resources and these calls for effectual network design and network planning. Call blocking probability, which defines the prospect of network services being deprived of to subscriber due to the nonavailability of radio resources, is the principal factor employed for efficient network design and network planning. Handed over calls are more sensitive to blocking than new calls since blocking causes forced termination of already established calls which becomes annoying.

When a subscriber makes a request for a handover, the network must assign resources to be used in routing the call to next base transceiver station. In situations where the handover fails to occur at the right time, the QoS drops below the set threshold level and the subscriber call is terminated and this constitute handoff call blocking. Other parameters involved in the handover decision include cell blocking probability, call completion probability, probability of unsuccessful handover, handover blocking probability, rate of handover, interruption duration and handover delay.

In their study, [3] explain that apart from unavailability of time or frequency channel, low level of system configuration and high traffic rates can also lead to call blocking. One of the salient features of GSM is movement of customers within the coverage area. These mobility patterns usually vary continuously due to factors such as rush hours, during which hot spots consisting of a bigger than average number of users travel along a highway. Consequently, this bigger number of subscribers brings forth a larger fresh call arrival rate.

These users making calls while in transit through the cellular network provoke handovers that occur when a call is relocated from the coverage area of a cell into the coverage area of a neighboring cell. It is possible for some subscribers whose handovers are blocked to attempt re-establishing their connection. Regrettably, so long as there are no free resources in the targeted cell, these redials are bound to be blocked, further adding to the call blocking probability.

\section{A. Teletraffic Models}

Teletraffic models have an ability of simulating real-life conditions and as such, a number of models have been created so as to approximate blocking probabilities that assist network engineers in dimensioning. As [4] discuss, blocking probabilities are affected by a number of factors such as call arrival patterns, number of servers or channels, channel holding time and traffic intensity. The Erlang B is the most preferred model for blocking probability computations throughout the world. However, this model was initially designed for fixed telephone networks and primarily trunks, although it has been ported to cellular network for the air interface dimensioning.

According to [5], Erlang B model was formerly employed in PSTN to dimension trunks, although it is nowadays usable in mobile telephone network dimensioning of the air interface. It is normally utilized in situations where subscribers do not perform call redials when their initial connections are blocked. The assumptions in this model are that the number of sources is unbounded, calls arrive arbitrarily and are served in the order in which they arrive, blocked calls are lost and call holding times are exponentially distributed. The Erlang $\mathrm{B}$ loss probability $\mathrm{P}_{\mathrm{B}}$ is given by eq. (1): 


$$
P_{B}=\frac{\left(\frac{A^{N}}{N !}\right)}{\sum_{i=0}^{N} \frac{A^{i}}{i !}}
$$

where $N$ and $A$ represent the number of channels available in resource group and traffic offered to group in

Erlangs, respectively.

In [6], it is noted that analysis of numerous parameters such as call arrival rate, call average holding time with various sample sizes and network resources have shown that Erlang B presents a good model for cells whose number of channels is larger than twelve, with blocking rate higher than $1 \%$. However, Erlang B is not suitable for GSM cells with less than twelve traffic channels. This is because in these circumstances, blocking probabilities are prone to overestimations.

Another teletraffic model is the Erlang $\mathrm{C}$, which is applicable in networks implementing call queuing instead of dropping them when all resources are in use [7]. Here, calls are put in a waiting queue until network resources are available or queue timer. Consequently, in the Erlang $\mathrm{C}$ model, blocked calls are delayed instead of being dropped immediately resource unavailability is detected. The probability that a customer will wait for service is given by eq. (2):

$$
P_{C}=\frac{\frac{A^{N} N}{N ! N-A}}{\sum_{i=0}^{N-1} \frac{A^{i}}{i !}+\frac{A^{N} N}{N ! N-A}}
$$

Where $\mathrm{A}$ is the total traffic offered in units of Erlangs, $N$ is the number of circuits and $P c$ is the probability that a customer has to wait for service. A close observation of eq. (1) and eq. (2) reveal that Erlang B and Erlang $\mathrm{C}$ are related as shown in eq. (3):

$$
P_{C}=\frac{N E_{B}}{N-A\left(1-P_{B}\right)}
$$

The setback of Erlang $\mathrm{C}$ is that when resources begin to be engaged, the blocking rate is higher. This can be attributed to the fact that this model takes into consideration the number of instances in the queue and the resources utilized.

\section{B. Call Blocking Triggers}

A number of factors have been observed to trigger call drop in a GSM cellular networks. These include radio frequency, handover failure, and low level of system configuration settings [8]. Radio frequency call blocking is occasioned by downlink and uplink failure. This is attributed to the fact that in the presence of numerous interferences in downlink and uplink, the mobile station cannot decode the slow associated control channel $(\mathrm{SACCH})$, which carries the system information message crucial for call connection. In circumstances where the mobile station is unable to decode $\mathrm{SACCH}$, it frees the radio channel connection abruptly, resulting in call drop. According to [9], radio frequency call blocking can also be brought about by feeble coverage area and radio signal, intra-network interference, deficiency of proper radio parameter settings, hardware malfunction and power collapse.

Handover failures on the other hand crop in when the mobile station receives a handover command but fails to execute the handover to its targeted cell, while at the same time it is unable to communicate with original cell. [10] discuss that in this scenario, the mobile station is detached from its network and the handoff control timer of base station informs the mobile switching center (MSC) about this failure, which then frees the channel. Afterwards, the MSC relinquishes the channels allocated to this mobile station and regards it as handover failure, effectively resulting in call disconnection. 


\section{Related Work}

The economic usefulness of any cellular network centers on the traffic that can be supported by the blocking probability. As such, the quality of service depends on the blocking probability estimation. In GSM and CDMA cellular systems, the blocking probability is determined by using the Erlang-B formula. Call blocking probability is one of the main criteria for GSM call handovers. As such, a number of researchers have developed and suggested different ideas and approaches that are believed to contribute to the reduction of handover dropping probability.

One of the approaches that have been developed for the reduction of handover failure rate and the resulting call drops is the prioritization of handover calls over new calls. As [11] explain, this strategy has a considerable influence on the call dropping probability and call blocking probability. Three variants of handover prioritization do exist and include guard channels, call admission control and handover queuing schemes.

In guard channel prioritization scheme, also referred to as cutoff priority schemes, a number of channels are reserved solely for handover in each cell. The rest of the channels are then shared evenly between handover calls and new calls [12]. Guard channel prioritization scheme is only applicable when the number of free channels is equal to or less than the predefined threshold. Consequently, the cell circumvents new calls and attends to only handover requests until all channels are engaged. This is ideal since handover calls are require continuity for voice and message transfer. On the other hand, call admission control prioritization scheme deals with the task of deciding whether fresh call requests should be permitted into the cellular network or denied access.

It involves continuous estimation of the arrival rate of new call and if this rate is higher than the set threshold level, some calls are blocked irrespective of whether a channel is available or not. In this scheme, both fresh and handover calls have access to all cell channels [13]. In situations where a fresh call is attempted but no idle channel is available, the call is dropped immediately as there are no queues offered for the new calls to be buffered.

According to [14], handover queuing prioritization schemes line up handover calls in the base station controller (BSC) when all the channels are occupied. First in first out (FIFO) scheme is the most preferred queuing scheme, in which the handover requests are prearranged in the order of arrival. Consequently, when a channel is freed in the BSC, it is allocated to one of the handover call requests in the queue.

In circumstances where the received signal strength in the current cell goes below a predefined threshold, the call is queued from serving a neighboring cell [15]. Unfortunately, this scheme lessens call dropping probability at the expense of increased call blocking probability and decline in the ratio of carried to admitted traffic. This is because fresh calls cannot be allocated a channel till such a time when all handover requests in the queue have been served.

The only time that a new call request is allocated a channel is when the queue is empty and there is a free channel in the BSC, otherwise the call remains queued until either a channel is freed in the target cell or the power of the base station in the current cell goes below the receiver threshold [16]. When the power sinks below the receiver threshold and all channels are busy, then the call is dropped. Handover queuing is feasible as a result of overlapping regions that exist between neighboring cells. At this region, the mobile station is able to communicate with more than one base station. On the flip side, [17] points out that this scheme is only effective when the handover requests appear in groups and teletraffic is at minimum.

In their paper, [5] proposed using auxiliary stations to reduce call blocking. Here, a base transceiver station contains two or more auxiliary stations such that whenever a call arrives at the base station, it is first served by an auxiliary station provided it has free channels till a channel is freed in the base station. When this happens, the call is transferred from the auxiliary station to the base station. For the duration during which the mobile station is within the auxiliary station, it sends out requests to the base station within predefined time intervals. In situations where any free channel is detected in base station, the mobile station will automatically connect 
with it, tearing down the connection to the auxiliary station. Although this process effectively decreases handoff failure probability, it can also lead to handoff failure when all the channels of adjacent auxiliary stations are busy.

In addition, call transfer time is needed when the closest auxiliary station has no free channels and call must be forwarded to second nearest auxiliary station. To overcome the problem of call dropping when all the auxiliary stations are bust, auxiliary stations can be allocated according to call durations. Here, one of the auxiliary stations is dedicated for serving long duration calls while the other auxiliary stations is dedicated for servicing short duration calls. However, if both auxiliary stations are busy and the base transceiver station has no free channels, then the received signal strength (RSS) of the mobile station is determined. The idea is that if the RSS is weak and the mobile station has been listed with neighboring cell, then mobile station connection request from auxiliary station is rejected.

A call blocking reduction scheme based on hierarchical dual-band cellular mobile communication networks has been proposed Here, both macro-cells and microcells are serviced by disparate base stations located at the center of the hierarchical network. The subscriber velocity is used as criteria of routing calls to either a macrocell or a microcell. Here, when the subscriber's velocity is very fast, the call is routed to the macro cell; else the call is serviced by the microcell.

On routing the call to the microcell, the needed bandwidth of this call is checked to establish whether it is larger than the available bandwidth, during which the call is re-routed to the macro cell. One setback of hierarchical cellular network is that call operation and signaling protocol for mobile terminals is sophisticated. In [18], a hybrid channel allocation, which is an amalgamation of fixed channel allocation and dynamic channel allocation is suggested as a possible solution to the call dropping problem.

Here, in circumstances where a subscriber requires a channel for the call and all the fixed set channels are busy, a request is made for a channel from the dynamic set. However, after the subscriber terminates a cal, the serving base station has to return the requested dynamic channels to the channels to mobile switching center, failure to which there will shortage of channels in dynamic set for further requests, resulting in calls being dropped. Bandwidth sharing through channel reuse between real time and non-real time handoff calls has been observed to reduce blocking probability [19]. Here, bandwidth is reserved in more than one cell, but this is not mandatory in circumstances where the subscriber's future location can be predicted with high accuracy.

\section{Basic Concepts in GSM Teletraffic Blocking}

A number of concepts come into play when synthesizing teletraffic blocking probabilities and they include GSM channel access, call request routing, traffic intensity and Poisson call arrival rates.

\section{A. GSM Channel access}

Each mobile cellular network operator is assigned a predefined restricted range of frequencies for the subscribers. As such, the capacity of the system in terms of the number of channels per BTS is also limited. Therefore, efficient channel utilization is the main issue for the enhancement of network performance [20]. Since the GSM cellular network makes use of the radio spectrum which is a very limited resource, the available bandwidth in second generation $(2 \mathrm{G})$ is divided using a combination of TDMA and FDMA. In third generation (3G) cellular systems, CDMA is employed while in fourth generation (4G) Orthogonal Frequency Division Multiple Access (OFDMA) technique is employed. The provision of high data rate transmission coupled with efficiency for high bandwidth, ability to operate in multipath radio environment and efficient sharing of limited resources are the salient features of OFDMA systems.

In [6], it is explained that for the case of FDMA cellular networks, the available frequency spectrum is partitioned among logical channels such that each subscriber has exclusive hold of some frequency band. Here, the maximum frequency bandwidth is $25 \mathrm{MHz}$ which is divided into 124 carrier frequencies, with each base 
transceiver station (BTS) being allocated one or more carrier frequencies. On the other hand, in TDMA systems, each carrier frequency is divided in time, forming logical channels. Each subscriber takes turn in channel utilization in a round robin manner, periodically accessing the full bandwidth for a little time slot. In CDMA cellular networks, each subscriber is allocated a disparate pseudorandom binary sequence for modulating the carrier, essentially scattering the spectrum of the waveform and assigning each subscriber a unique code pattern.

\section{B. Service Request Routing}

In cellular networks, before a traffic channel for a set-up request can be assigned, the subscriber request goes first through the random access channel $(\mathrm{RACH})$ and thereafter through the stand-alone dedicated control channel $(\mathrm{SDCCH})$. After these two channels are effectively utilized, the subscriber's identity is verified and further resources can be allocated on request.

When the capacity of RACH and SDCCH is fully utilized, further call requests are rejected. Any call reties will add up to the blocking probability as they are treated as fresh call requests [13]. Since access to SDCCH is required in order to utilize the Short Message Service (SMS), a high blocking probability on SDCCH deters access to all GSM services.

\section{Traffic Intensity}

Traffic intensity is linked to the product of average call duration and the average number of call requests [6]. It is measured in Erlangs and when one radio channel resource is utilized for one hour, one Erlang of traffic is said to have flown through. Mathematically, this can be represented as shown in eq. (4):

$$
A_{C}=\tau h
$$

Where $A_{C}$ is the traffic intensity in Erlangs generated by each subscriber, $h$ is the average call holding time measured in hours and $\tau$ is the average number of call requests per hour.

\section{Poissonian Call Arrival Rates}

The Poisson distribution model depicts the likelihood of a given number of events taking place in a fixed interval of time given that these events transpire with an established constant rate and independently of the time since the last event took place. According to [21] four assumptions are critical for the application of the Poisson distribution: given that $n$ is the number of times an event takes place in an interval $\Delta t$, then $n$ can take only positive integer values; the happening of one event does not have an effect on the possibility that a second event will take place, meaning that these events occur independently; the rate at which events takes place is constant, meaning that this rate cannot be higher in some intervals and lower in some other intervals; two events cannot happen at exactly the same instant, hence at each very small sub-interval, exactly one event either occurs or fails to take place at all; the likelihood of an event in a small sub-interval is proportional to the length of the sub-interval. Mathematically, the probability mass function for a Poisson distribution can be expressed as shown in eq. (5):

$$
P(k \text { events in } \Delta t)=e^{-\tau} \frac{\tau^{n}}{n !}
$$

Where $\tau$ is the average number of events occurrence per interval, $e$ Euler's number, representing the base of the natural logarithms and its value is approximately $2.71828, n$ is the number of times an event takes place in an interval $\Delta t$, and $n$ ! is the factorial of $n$. 


\section{Methodology}

In this paper, GSM cellular network traffic is synthesized taking into consideration both RACH and SDCCH channels. The traffic considered here includes both voice and data and it is assumed that during an overload situation, all traffic channels are busy all the time. As such, each request attempt has a very low possibility of finding a free traffic channel. The arrival rates are taken to be exclusively independent. Fig. 1 shows the signal flow representation employed in this paper. As shown in Fig. 1, apart from the normal services requests, users can make retries and it is also possible for the requests to experience timeouts and collisions. In addition, there is need for a channel for RACH request acknowledgement.

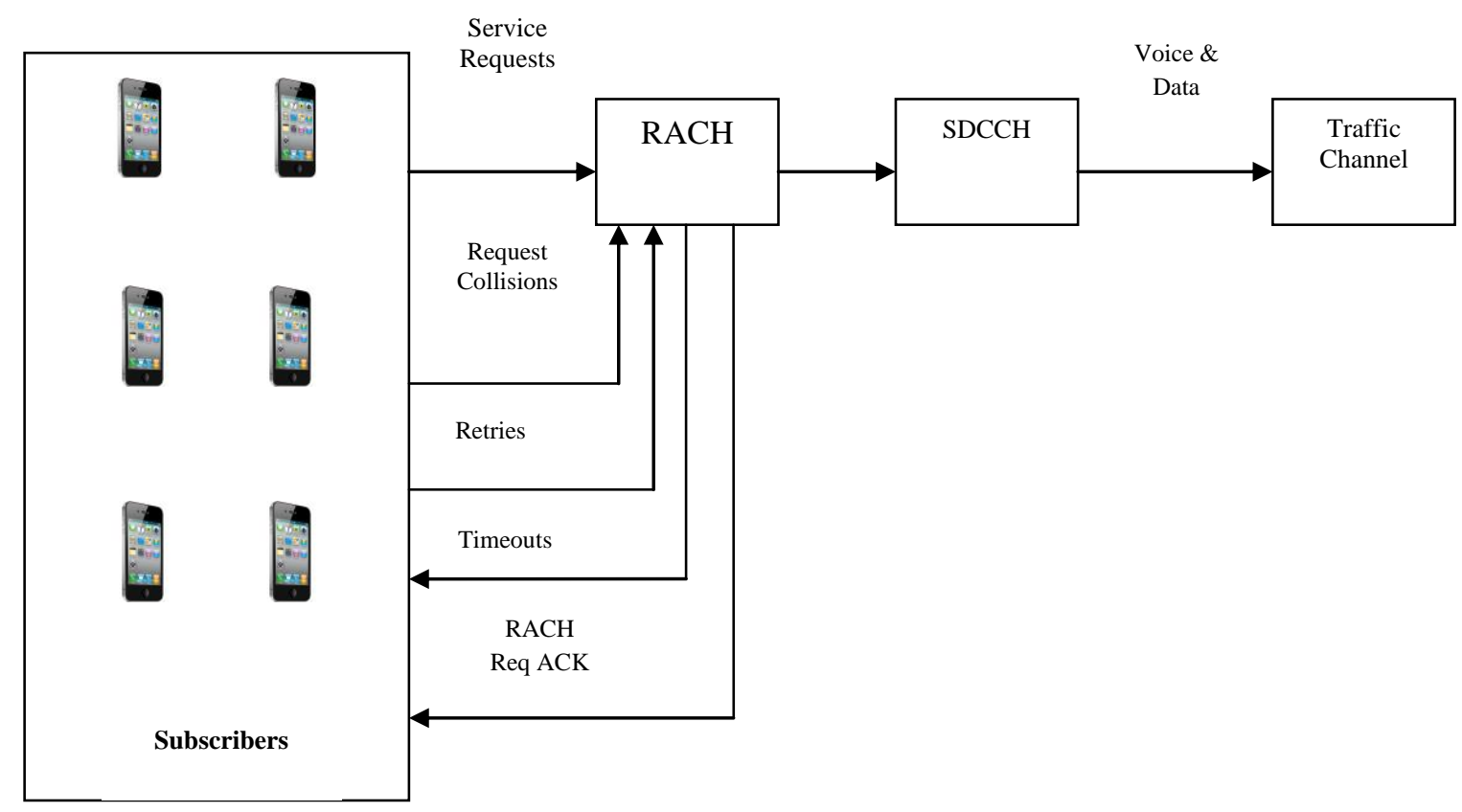

Fig.1. GSM Signal Flow

\section{A. Fitting Poissonian Distribution to GSM Networks}

The call arrival rates on a GSM BTS can be regarded as a Poisson process, a commonly used model for random, mutually independent events. The four assumptions explained above can be fitted to the GSM network as follows: $n$, which is the number of times an event takes place in an interval $\Delta t$, can be taken as the number of times subscribers make fresh call attempts or handover requests and hence $n$ can take only positive integer values; the property that the happening of one event does not have an effect on the possibility that a second event will take place can be taken to mean that a call attempt or handover request made by one subscriber does not affect the probability of another subscriber making a call attempt or handover request, meaning that subscriber call attempts are mutually exclusive; the two events that cannot happen at exactly the same instant can be taken to be successful call attempt or handover request that cannot take place at exactly the same instant as a failed call attempt or handover request, it is either a success or a failure but not both; the likelihood of an event in a small sub-interval which is proportional to the length of the sub-interval can be taken to mean the 
probability of a successful call attempt or handover request which is proportional to the number of channel present in a GSM network.

Consequently, the Poisson distribution model perfectly fits into the cellular network call and handover request arrival synthesis. The only assumption that does not apply is that the rate at which events takes place is constant, because in GSM network, at peak hours, more call and handovers arrive at the BTS compared to offpeak hours. If the mean time between call arrivals is $\frac{1}{\Delta t}$ and the mean call holding time is $\frac{1}{h}$, then the traffic to the GSM network is given by eq. (6):

$$
A_{G S M}=\frac{\Delta t}{h} \text { Erlangs }
$$

Taking $N$ to be the random variable representing the number of active calls in a GSM network at steady state, then, $N$ has the Poisson distribution given by eq. (7):

$$
P(N=n)=e^{-A_{G S M}} \frac{\left(A_{G S M}\right)^{n}}{n !}
$$

The independence of each fresh call attempt or handover request in each BTS in the network has the implication that the joint steady-state distribution for the number of active calls in each BTS is basically a product of Poisson distributions.

\section{B. Teletraffic Blocking Synthesis within Lossy GSM Network}

A number of losses occur in GSM networks. These include losses due to request time outs, collisions of subscriber for RACH capacity and call loss as a result of SDCCH channel deficiency. Taking these losses into consideration, Fig. 1 can be modified to yield Fig. 2. Here, $\tau$ is the call arrival rate, $\eta$ is the subscriber RACH capacity services request collisions and $E$ is the SDCCH channel deficiency losses.

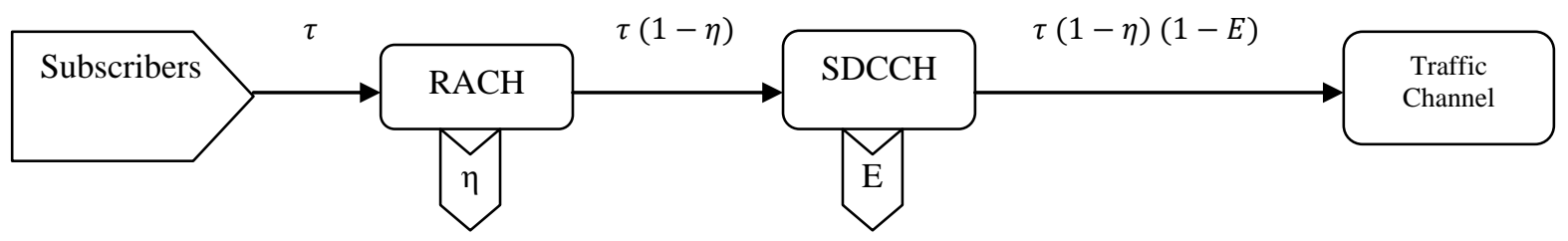

Fig.2. Teletraffic Blocking Synthesis within Lossy GSM Network

\section{RACH Based GSM Teletraffic Blocking Probabilistic Synthesis}

In this situation, three parameters are employed to compute the RACH effective service request rate $\left(R_{A C H}\right)$. These include the fresh calls arrival rates as initiated by subscribers, the likelihood that a subscriber's service request is colliding with other subscribers' requests and therefore not able to be serviced by the RACH capacity, and the number of retries. Therefore, the RACH effective service request rate is given by eq. (8):

$$
R A C H_{e f f}=\tau \sum_{i=0}^{n-1}(i+1) \eta^{i}(1-\eta)+(n+1) \tau \eta^{n}=\tau \sum_{i=0}^{n} \eta^{i}=\tau \frac{1-\eta^{n+1}}{1-\eta}
$$


where $\tau$ is the fresh calls arrival rate, $\eta$ is the services request collision probability and $n$ is the number of retries.

Assuming no retries are permitted and taking $N$ to be the number of subscribers each with a probability of attempting a new call in a RACH time slot $t$, the success access probability is given by eq. (9):

$$
R A C H(\text { retries }=0)_{\text {eff }}=N t(1-t)^{N-1}
$$

Considering only a specific subscriber, the probability in eq. (9) reduces to:

$$
R A C H(\text { specific sub })_{e f f}=(1-t)^{N-1}
$$

This means that a fresh call attempt can only be successful when none of the subscribers are transmitting in the same slot. Clearly, call retries add to the effective call attempt rates. The resultant likelihood that no other transmissions both new and retry is initiated in the same time slot as a particular attempt can be estimated by eq. (11):

$$
e^{-R A C H_{e f f}}
$$

Comparing eq. (4) with the probability mass function for a Poisson distribution of eq. (5), it is clear that eq. (4) has introduced some Poissonian arrival model for fresh call requests. This can be attributed to the large number of subscribers each having a definite request, hence generating probability for that particular time slot. As such, the services request collision probability is depicted by eq. (12):

$$
\eta=1-e^{-R A C H} e f f
$$

The probability that a particular subscriber's fresh call attempt gets through is then given by eq. (13):

$$
\text { Sub } b_{\text {fresh call }}=1-\eta^{n+1}
$$

Where $\eta=1-e^{-R A C H_{e f f}}$

\section{SDCCH Based GSM Teletraffic Blocking Probabilistic Synthesis}

In GSM networks, the performance of the SDCCH depends on the actual call arrival rates and the equivalent call holding times. In this synthesis, losses due to lack of RACH will be assumed to be negligible. The call loss probability as a result of SDCCH channel deficiency can be estimated by the use of Erlang's formula. Here, the carried traffic rate provided to common voice channels can be estimated by eq. (14):

$$
S D C C H_{\text {voice }}=\tau(1-\eta)\left\{1-E_{1, S}\left(\tau(1-\eta) t_{S D C C H}\right)\right.
$$

where $t_{S D C C H}$ is the mean holding time of a dedicated control channel.

\section{E. GSM Teletraffic Blocking Probabilistic Synthesis With Service Request Retries}

In this synthesis, a fixed retry probability $p_{\text {ret }}$ which is independent of the number of retries a subscriber has made is assumed. As such, the resultant service request input rate (Service rate $_{\text {) }}$ ) is increased to: 


$$
\text { Service }_{\text {rate }}=\frac{\tau}{1-p_{\text {ret }}}
$$

Consequently, the input rate can be adjusted by just varying the subscriber call redials. The worst case is during heavy overload scenarios where the number of call redials is high and due to blocked calls and hence leading to an increase in the overall services input rates.

\section{F. GSM Teletraffic Blocking Probabilistic Synthesis in Overlay Cells}

An overlay cell consists of an umbrella cell, a macro cell servicing high velocity moving subscribers (HVMS) and a microcell serving low velocity subscribers (LVMS) as shown in Fig. 3. Umbrella and macro-cells have high transmit power, have their antennas above rooftop levels, provide large area coverage and serve mostly high velocity subscribers. On the other hand, microcells and pico-cells have low transmit power, have their antennas below rooftop levels or indoor (pico-cells), provide small coverage and serve low velocity subscribers.

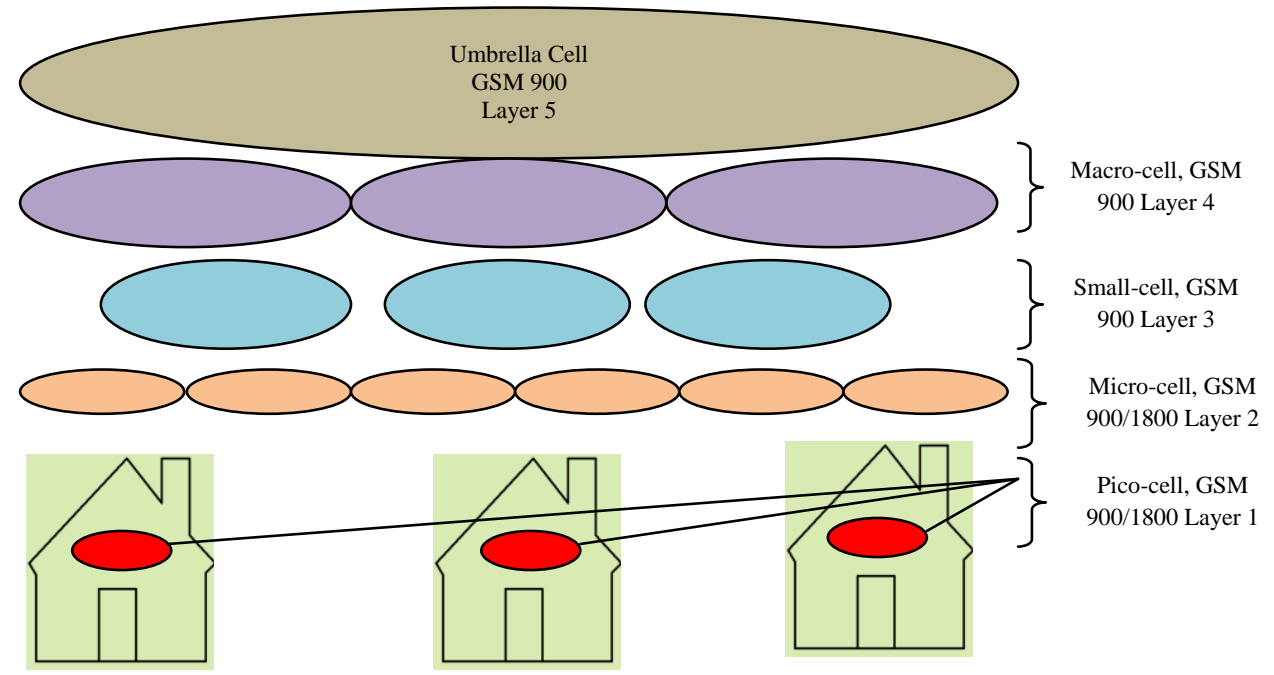

Fig.3. GSM Overlay Cellular Network

Assuming that the network implements some prioritization for handover calls, let $\mathrm{C}$ be the total channels in every microcell, and $C_{h}$ be the guard channels exclusively reserved for handover. This means that the remaining channels, given by $C-C h$ are shared equally by new and handover calls. Further, let traffic be homogenous in all microcells with same capacity and same mean holding time $(h)$.

Let the mean rate $(\tau)$ of new and handover calls of LVMS in a microcell generated by Poisson point process be $\tau_{N}^{S}$ and $\tau_{H}^{S}$ respectively. Moreover, let new and handover calls of HVMS have mean rates of $\tau_{N}^{F}$ and $\tau_{H}^{F}$ per cell. With these assumptions in mind, the relative mobility for HVMS is $R M_{(H V M S)}$ while the relative mobility for LVMS is $R M_{(L V M S)}$ as expressed in eq. (16): 


$$
\left\{\begin{array}{l}
R M_{(L V M S)}=\frac{\tau_{H}^{S}}{\tau_{H}^{S}+\tau_{N}^{S}} \\
R M_{(H V M S)}=\frac{\tau_{H}^{F}}{\tau_{H}^{F}+\tau_{N}^{F}}
\end{array}\right.
$$

The total relative mobility (RM) for both LVMS and HVMS is then given by eq. (17):

$$
R M_{(L V M S, H V M S)}=\frac{\tau_{H}^{F}+\tau_{H}^{S}}{\tau_{H}^{F}+\tau_{N}^{F}+\tau_{H}^{S}+\tau_{N}^{S}}
$$

Consequently, the total offered load (TOL) per cell is can be expressed by eq. (18):

$$
T O L_{\text {cell }}=\frac{\tau_{H}^{F}+\tau_{N}^{F}+\tau_{H}^{S}+\tau_{N}^{S}}{\mu_{C h}}
$$

Where:

$$
\mu_{C h}=\frac{1}{\text { Channel holding time }\left(T_{C h}\right)}
$$

Taking $n$ to be the number of microcells in a given microcellular region, the total offered load in the microcellular region is given by eq. (20):

$$
\text { TOL } L_{\text {Region }}^{\text {icrocellular }}=n . T O L_{\text {cell }}
$$

Recalling that $\mathrm{C}$ is the total number of channels in every microcell, then total number of channels in the microcellular region is depicted by eq. (21):

$$
C_{(\text {Microcelluar })}=n \cdot C
$$

Let us consider an off peak duration, during which all channels may be available in every microcell. In this situation, the probability $\left(P_{\text {offpeak }}\right)$ of occupying a channel by a new subscriber is given by eq. (22):

$$
P_{(\text {offpeak })}=\left[\sum_{i=0}^{C-C_{h}} \frac{\left(\tau_{H}^{F}+\tau_{N}^{F}+\tau_{H}^{S}+\tau_{N}^{S}\right)^{i}}{i ! \mu_{c h}^{i}}+\sum_{i=C-C_{h}+1}^{C} \frac{\left(\tau_{H}^{F}+\tau_{N}^{F}+\tau_{H}^{S}+\tau_{N}^{S}\right)^{C-C_{h}}\left(\tau_{H}^{F}+\tau_{H}^{S}\right)^{i-C+C_{h}}}{i ! \mu_{c h}^{i}}\right]^{-1}
$$

Now suppose that some subscribers are active in every cell and as a result, $a$ channels are active in each microcell. In this scenario, the steady state probability can be derived as shown in eq. (23):

$$
P_{a}=\frac{\left(\tau_{H}^{F}+\tau_{N}^{F}+\tau_{H}^{S}+\tau_{N}^{S}\right)^{a}}{a ! \mu_{c h}^{a}} P_{(\text {offpeak })}
$$

Where $\mathrm{a}=1,2, \ldots C-C_{h}$ 
Again:

$P_{a}=\frac{\left(\tau_{H}^{F}+\tau_{N}^{F}+\tau_{H}^{S}+\tau_{N}^{S}\right)^{C-C_{h}}\left(\tau_{H}^{F}+\tau_{H}^{S}\right)^{a-C+C_{h}}}{a ! \mu_{c h}^{a}} P_{(\text {offpeak })}$

For a $=C-C_{h}+1, \ldots \ldots, C$

Logically, the blocking probability $\left(P\left({ }_{H V M S, L V M S}\right)\right.$ for a new call initiated by either by HVMS or LVMS is the sum of probabilities that the state number $a$ of the microcell $\geq(C-C h)$. This can be expressed mathematically as shown in eq. (25):

$$
P_{(H V M S, L V M S)}=\sum_{a=C-C_{h}}^{C} P_{a}
$$

Intuitively, the likelihood of handover failure $(P($ Handoff failure $))$ is equivalent to the probability of the microcell with the state number $C$ as given in eq. (26):

$$
P_{(\text {Handoff failure })}=P_{C}
$$

As such, the handover failure probability for HVMS can be expressed by eq. (27):

$$
P_{(\text {Handoff failure })}^{H V M S}=\left(\frac{\tau_{H}^{F}}{\tau_{H}^{F}+\tau_{H}^{S}}\right) P_{(\text {Handoff failure })}
$$

In conclusion, for all the microcells within a microcellular region, when fresh and handover calls of LVMS and HVMS are taken into consideration, then the average call blocking probability is given by eq. (28):

$$
P_{(\text {average })}=\sum_{k=1}^{n} \frac{\left[\left(\tau_{N}^{F}(k)+\tau_{N}^{S}(k) P_{(H V M S, L V M S)}(k)+\left(\tau_{H}^{F}(k)+\tau_{H}^{S}(k) P_{(\text {Handoff failure })}(k)\right]\right.\right.}{\left[\tau_{H}^{F}(k)+\tau_{N}^{F}(k)+\tau_{H}^{S}(k)+\tau_{N}^{S}(k)\right]}
$$

Suppose the total channel capacity of an overlay network consisting of macro-cells and microcells is $C_{\text {(overlay) }}$ and $n$ is the number of microcells in the microcellular region. Further, let us assume that handover request are prioritized in this microcellular region by being allocated guard channels $C_{(\text {guard })}$ exclusively for handover calls of LVMS among the $C$ channels in a microcell cell. Consequently, the rest of the channels given by $C-C_{(\text {guard })}$ are equally shared by both fresh call attempts of HVMS and LVMS and handed over calls of LVMS. Further, let us assume that $C_{(\text {umbrella })}$ channels are assigned to umbrella cell to service only handed over calls of HVMU. Taking all these assumptions into consideration, the Channel Capacity of the overlay network is defined by eq. (29):

$$
C_{(\text {overlay })}=n \cdot C+C_{(\text {umbrella })}
$$

As already been demonstrated, the mean rate of handover calls of HVMS is $\tau_{H}^{F}$ per cell. As such, the mean rate created in the umbrella cell by HVMS is $n . \tau_{H}^{F}$, where $n$ is the total number of microcells.

In an effort to reduce the blocking probability of handover calls of HVMS in this network, it is appropriate to compute the ratio between $C_{(\text {umbrella) }}$ and $C_{(\text {overlay })}$. To accomplish this, values of $R M_{(L V M S)}, R M_{(H V M S)}$, $R M_{(L V M S, H V M S)}$ and TOL Region Microlular.

Suppose that it is an off-peak duration in which all channels may be available in every microcell. In this scenario, the probability $\left(P_{\text {Offpeak }}^{\text {micro }}\right)$ of occupying a channel by a new subscriber is given by eq. (30): 


$$
P_{\text {offpeak }}^{\text {micro }}=\left[\sum_{i=0}^{C-C_{(\text {guard })}} \frac{\left(\tau_{N}^{F}+\tau_{H}^{S}+\tau_{N}^{S}\right)^{i}}{i ! \mu_{C h}^{i}}+\sum_{i=C-C_{(\text {guard })}^{C}+1}^{C} \frac{\left(\tau_{N}^{F}+\tau_{H}^{S}+\tau_{N}^{S}\right)^{C-C_{(\text {guard })}\left(\tau_{H}^{S}\right)^{i-C+C_{(\text {guard })}}}}{i ! \mu_{C h}^{i}}\right]^{-1}
$$

Assuming that some subscribers are active, consuming $m$ number of channels, the steady state probabilities in a microcell becomes:

$$
P_{m}^{m i c r o}=\frac{\left(\tau_{N}^{F}+\tau_{H}^{S}+\tau_{N}^{S}\right)^{m}}{m ! \mu_{c h}^{m}} P_{o f f p e a k}^{\text {micro }}
$$

Where $m=1,2, \ldots ., C-C_{(\text {guard })}$

Also:

$$
P_{m}^{\text {micro }}=\frac{\left(\tau_{N}^{F}+\tau_{H}^{S}+\tau_{N}^{S}\right)^{C-C_{(\text {guard })}}\left(\tau_{H}^{S}\right)^{m-C+C_{(\text {guard })}}}{m ! \mu_{c h}^{m}} P_{\text {new }}
$$

for $m=C-C_{(\text {guard })}+1, \ldots \ldots, C$

In this setup, for any HVMS or LVMS per microcell, the blocking probability of a fresh call is obtained either by the summation of probabilities such that the static number of the microcell is larger than or equal to $\left(C-C_{(\text {guard })}\right)$. As such:

$$
P_{\text {blocking }}^{\text {micro }}=\sum_{m=C-C_{(\text {guard })}^{C}}^{C} P_{m}^{\text {micro }}
$$

Within a microcell, the probability of handover failure $\left(P_{(\text {Hofailure })}^{\text {micro }}\right)$ is equivalent to the probability of the microcell with the static number $C$, in which case:

$$
P_{(\text {HOfailure })}^{\text {micro }}=P_{C}^{\text {micro }}
$$

Suppose that subscribers are active within an umbrella cell, utilizing $k$ channels within this cell. Then, the steady-state probabilities can be expressed by eq. (35):

$$
P_{k}^{u m b r e l l a}=\frac{\left(n \cdot \tau_{H}^{F}\right)^{k}}{k ! \mu_{c h}^{k}} P_{O}^{u m b r e l l a}
$$

For $m=1,2, \ldots \ldots C_{(\text {umbrella })}$

Where:

$$
P_{O}^{\text {umbrella }}=\left[\sum_{i=0}^{C_{(u m b r e l l a)}} \frac{\left(n \cdot \tau_{H}^{F}\right)^{i}}{i ! \mu_{c h}^{i}}\right]^{-1}
$$

Notably, it comes out clearly that probability of handover failure in umbrella cell is equivalent to the probability with the static number $C_{(\text {umbrella) }}$ number of cells as given in eq. (37):

$$
P_{(\text {Hofailure })}^{\text {umbrella }}=P_{C_{(\text {umbrella })}}
$$


As such, the average call blocking probability for the case of a microcellular layer with $n$ microcells, in the presence of the fresh calls of LVMS and HVMS and handover calls of LVMS is:

$$
P_{(\text {average })}^{\text {microcellular }}=\sum_{k=1}^{n} \frac{\left(\left(\tau_{N}^{F}(k)+\tau_{N}^{S}(k)\right) P_{\text {blocking }}^{\text {micro }}(k)+\left(\tau_{H}^{S}(k)\right) P_{(\text {HOfailure })}^{\text {micro }}(k)\right)}{\left(\tau_{N}^{F}(k)+\tau_{H}^{S}(k)+\tau_{N}^{S}(k)\right)}
$$

From eq. (37), the average call blocking probability within a microcellular region takes into consideration of the high velocity subscribers, low velocity subscribers, overlay cells as represented by handover calls of low velocity mobile subscribers (LVMS). This is superior to both Erlang B and Erlang C models which only consider the number of channels available in resource group and traffic offered to group.

\section{G. GSM Teletraffic Blocking Probabilistic Synthesis with Queue Delays}

In this section, the call blocking probability is synthesized, taking into consideration factors such as Rayleigh fading, Poisson distribution of service demand, first-in first-out (FIFO) queues and fixed length queues. Rayleigh fading happens when the received signals are exposed to multiple reflections, in most cases distorted through walls and other obstruction which causes these signals to sometimes combine out of phase, effectively cancelling each other. In this case, the probability of a call delay due to surging demand in the Poisson process can be expressed by eq. (39):

$$
P_{\text {delay }}=\frac{(N)\left(P_{B}\right)}{N-A_{\text {offered }}\left(1-P_{B}\right)}
$$

Where $N$ is the number of available channels, $P_{B}$ is the Erlang-B formula and $A_{\text {offered }}$ is the offered teletraffic.

In circumstances where this delay is larger than the network queue length $\left(Q_{L}\right)$, then the call is blocked with probability $\left(P\left(\right.\right.$ Delay $\left.>Q_{L}\right)$ given by eq. $(40)$ :

$$
P\left(\text { Delay }>Q_{L}\right)=P_{N+Q_{L}}\left(A_{\text {offered }}\right)=\frac{P_{H D}\left(A_{\text {offered }}\right) A_{\text {offered }}{ }^{N+Q_{L}}}{N ! N^{Q_{L}}}
$$

Where $P_{H D}$ is the probability of high demand and $Q_{L}$ is the queue length.

\section{H. Teletraffic Blocking Probabilistic Synthesis with Markov Chain Cell Occupancy}

In this part, a consideration is given to a cellular network with total number of channels in a given cell being equal to $N$. Suppose that a channel reservation scheme is applied that keeps $N-P_{T}$ channels for handover are where $P_{T}$ is the predefined threshold. Consequently, this network will not accept any fresh calls until the channel occupancy is well below the threshold. Taking the arrival of fresh and handover calls to be $\gamma$ and $\psi$ respectively, and the call holding and call residency for both fresh and handover calls to be exponentially distributed with $\frac{1}{\alpha}$ and $\frac{1}{\beta}$ respectively, the total teletraffic $\left(A_{T}\right)$ can be expressed as:

$$
A_{T}=\frac{(\gamma+\psi)}{(\alpha+\beta)}
$$

As such, according to the cell occupancy by Markov chain, it is easy to derive the steady state probability $P_{\text {busy }}$ that $n$ channels are busy as follows: 


$$
P_{\text {busy }}=\left\{\begin{array}{c}
\left(\frac{A_{T}^{n}}{n !}\right) P_{0}, 0 \leq n \leq P_{T}, \\
A_{T}^{P_{T}}\left(\frac{\psi^{n-P_{T}}}{n !}\right) P_{0}, P_{T} \leq n \leq N
\end{array}\right.
$$

Where:

$$
P_{0}=\left[\sum_{n=0}^{P_{T}} \frac{A_{T}^{n}}{n !}+A_{T}^{P_{T}} \sum_{n=P_{T}+1}^{N} \frac{\psi^{n-P_{T}}}{n !}\right]^{-1}
$$

In FIFO scheme, handover requests are ordered according to their arrival time. Suppose priority is offered for handoff requests by assigning $C_{(\text {guard })}$ channels exclusively for handoff calls out of $C$ channels in a cell. In this case, both new calls and handoff requests share the remaining $C_{\text {shared }}$ channels, where $C_{\text {shared }}=C-$ $C_{(\text {guard })}$. Here, a fresh call will be blocked when the available number of channel are $\leq C_{\text {shared }}$. This can be represented as shown in Fig. 4.

Let $k(k=0,1,2, \ldots . C)$ be the state of a cell representing the number of calls in progress for the BTS of that cell shown in Fig. 5. Suppose that $P(k)$ is the steady-state probability that the BTS is in state $k$, then the probabilities $P(k)$ can be expressed for birth-death processes as shown in eq. (44).

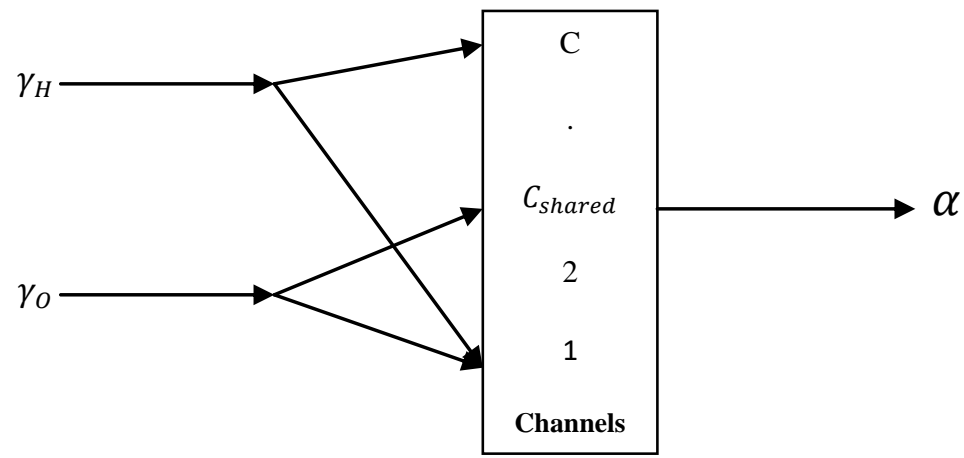

Fig.4. Teletraffic Blocking with Markov Chain Cell Occupancy

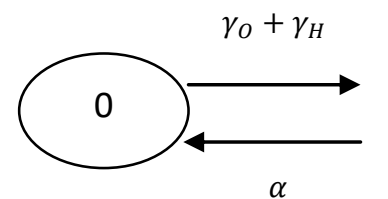

$\alpha$

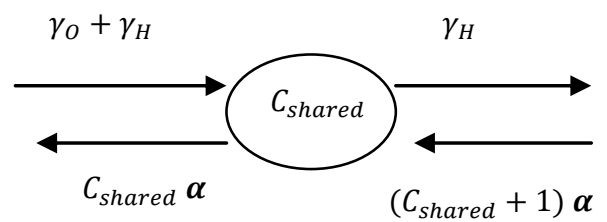

$\left(C_{\text {shared }}+1\right) \alpha$

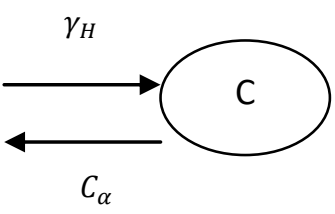

Fig.5. State Transition Diagram for Prioritized Handover

$$
P(k)=\left\{\begin{array}{c}
\frac{\left(\gamma_{O}+\gamma_{H}\right)^{k}}{k ! \alpha^{k}} P(0) 0 \leq k \leq C_{\text {shared }} \\
\frac{\left(\gamma_{O}+\gamma_{H}\right)^{C} \text { shared } \alpha_{H}^{k-C_{\text {shared }}}}{k ! \alpha^{k}} P(0) C_{\text {shared }} \leq k \leq C
\end{array}\right\}
$$


Based on eq. (43) the steady state probability $P(0)$, which describes the possibility that the network is in state $[0]$ can be expressed as shown in eq. (45):

$$
P_{(0)}=\left[\sum_{k=0}^{C_{\text {shared }}} \frac{\left(\gamma_{O}+\gamma_{H}\right)^{k}}{k ! \alpha^{k}}+\sum_{k=C_{\text {shared }}+1}^{C} \frac{\left(\gamma_{O}+\gamma_{H}\right)^{C} \text { shared } \alpha_{H}^{k-C_{\text {shared }}}}{k ! \alpha^{k}}\right]^{-1}
$$

Essentially, the blocking probabilities $P_{\text {fresh }}$ for a fresh call and $P_{H O}$ of a handoff request can be expressed as shown in eq. (46) and eq. (47) respectively:

$$
P_{\text {fresh }}=\sum_{k=C_{\text {shared }}}^{C} P_{(k)}
$$

And:

$$
P_{H O}=P_{(C)}=\frac{\left(\gamma_{O}+\gamma_{H}\right)^{C} \text { shared } \alpha_{H}^{C-C_{\text {shared }}}}{C ! \alpha^{C}} P_{(0)}
$$

Important to note is that a subscriber whose handoff request is blocked can still sustain the communication using either the present BTS until the conversation is concluded before the received signal strength goes below the receiver threshold. In addition, in the FIFO handover prioritization scheme, the prospect of the forced termination is reduced, although the handover request can still be dropped since the handover request can only wait until the receiver threshold is reached.

\section{Teletraffic Blocking Probabilistic Synthesis in OFDMA Cellular Networks}

In orthogonal frequency division multiple accesses (OFDMA) cellular networks, orthogonal subcarriers are allocated to subscribers based on their service rate requirement. This again depends on the signal to interference ratio (SIR) experienced by the individual subscribers. Because of this, the blocking probability analysis of conventional cellular systems such as GSM is not applicable in cellular OFDMA. Based on the channel capacity theorem, the data rate of OFDMA can be attained by assigning the $i^{\text {th }}$ subcarrier to subscriber as:

$$
R=\log _{2}\left(1+S I N R_{i}\right)
$$

Where $\mathrm{R}$ is the cell radius and SINR is the signal to interference noise ratio which at reference cell for $i^{\text {th }}$ subcarrier is given by eq. (49):

$$
\operatorname{SINR}_{i}=\frac{P_{t x} d^{-\eta} 10^{\frac{\varepsilon}{10}}}{I_{i}+N_{0}}, \quad 1<i<N
$$

Where $P_{t x}$ is the power transmitted by the BTS on the $i^{t h}$ subcarrier, $d$ is radial distance between subscriber and the BTS, $\eta$ is the exponent of path-loss, $\varepsilon$ is the shadowing effect, $N_{0}$ is the thermal noise density and $I_{i}$ is the interference received on $i^{\text {th }}$ subcarrier.

In OFDMA, the interference received on $i^{\text {th }}$ subcarrier $\left(I_{i}\right)$ is given by eq. (50):

$$
I_{i}=\sum_{j=1}^{6} I_{i, j} l_{A}(i, j)
$$


Where $I_{i, j}$ is the interference from $j^{\text {th }}$ neighboring cell $1 \leq j \leq 6, d_{j}$ is the distance between subscriber in the reference cell and subscriber in the $j^{\text {th }}$ neighboring cell, $l_{A}(i, j)=1$ when $i^{\text {th }}$ subcarrier is assigned to mobile station of $j^{t h}$ neighboring cell, $l_{A}(i, j)=0$ otherwise. Suppose that a subscriber call has arrived in the reference cell, upon which the base station assigns a set of subcarriers to this call. In this scenario, this incoming call is blocked if number of subcarriers available at the BTS is less than the number of subcarriers required for this subscriber. This blocking condition $\left(P_{O F D M A}\right)$ is given by eq. (51):

$$
P_{\text {OFDMA }}=\sum_{i=1}^{n_{\text {sub }}} \log _{2}\left(1+S I N R_{i}\right) \geq s u b_{\text {req }}
$$

Where $s u b_{r e q}$ is the rate required by the subscriber, and $n_{s u b}$ is the number of subcarriers required by the subscriber.

\section{Numerical Results}

To validate the GSM blocking probabilities derived in section III above, a number of scenarios were run and observations made. This included an investigation on the effect of the arrival rates of subscribers on Poission probability distribution, effect of call holding time on traffic intensity, effect of BTS channels capacity on blocking probability, impact of offered traffic on blocking probability, influence of subscriber distribution density on blocking probability, and the effects of queued traffic on handover blocking probability. For all the simulations, it was assumed that the subscriber arrival rate was a Poisson process and the outcomes of these simulations are presented in the subsections that follow.

\section{A. Effect of Varying Arrival Rates on Poissonian Probability Distribution}

To investigate the effect of the arrival rate of subscribers at a BTS, 30 subscribers were considered and this effect was computed using Poissonian distribution model. The results obtained are shown in Fig. 6.

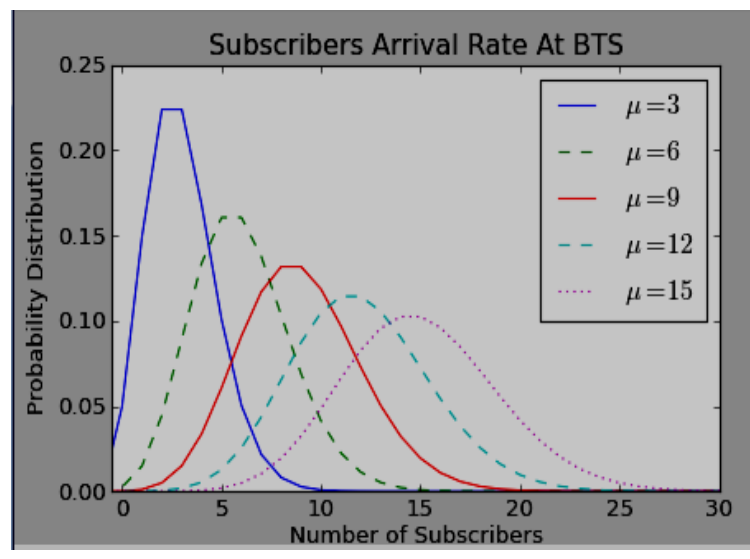

Fig.6. Probability Distribution for varying Arrival rates

As Fig. 6 shows, as the value of subscriber arrival rate increases, the shape of the probability distribution shifts dramatically to a more symmetrical and normal form. As an illustration, when the subscriber arrival rate is increased from 3 to 15 , the shape of the graph is that of a normal distribution. It was also noted that the probability of a larger number of arrivals increased with souring number of subscribers. The rapid arrival processes of subscribers provide larger contact probabilities. This can be attributed to the subscribers' contiguity to one another, rendering mobility of these subscribers difficult. 
In an actual GSM cellular network, subscribers can arrive at a BTS either in a regular pattern or in a random pattern. The subscriber arrival rates have an influence on the number of mobile stations in a particular region, even though each region or cell has a restricted capacity. As the number of mobile stations increase, the average number of neighbors per mobile station surges and these impacts on route availability. Additionally, the total connection time of a mobile station over a specific interval of time depends on the subscriber's call initiation rate and the call holding time, both of which depend on the relative mobility of subscribers. A high subscriber arrival rate causes more mobile station to be located in a particular region, and hence the probability of them initiating high number of calls, rendering the network congested. The effect of this correlation is that mobile stations can be tightly packed, experiencing high density when their arrival rate is high, leading to congestion. On the contrary, when their arrival rate is lesser, the mobile stations are located farther apart, hence experiencing low density.

\section{B. Effect of Call Holding Time On Traffic Intensity}

In this section, the number of call and handover request were varied from 1 to 9 per hour while the holding time was increased from 10 to 15 minutes, translating to 0.167 to 0.25 holding time per hour. Table 1 shows the results obtained.

Table 1. Variation of Traffic Intensity with Number of Request

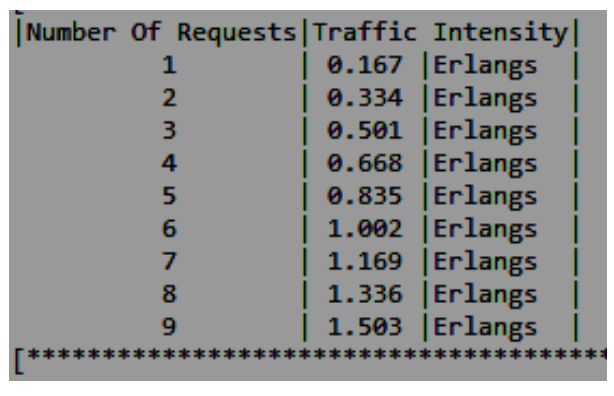

As Table 1 shows, as the number of call requests were increased from 1 to 9 , the traffic intensity generated is also increased from 0.167 Erlangs to 1.503 Erlangs. Fig. 7 that follows gives the results obtained when the subscriber holding time was varied between 10 and 15 per hour.

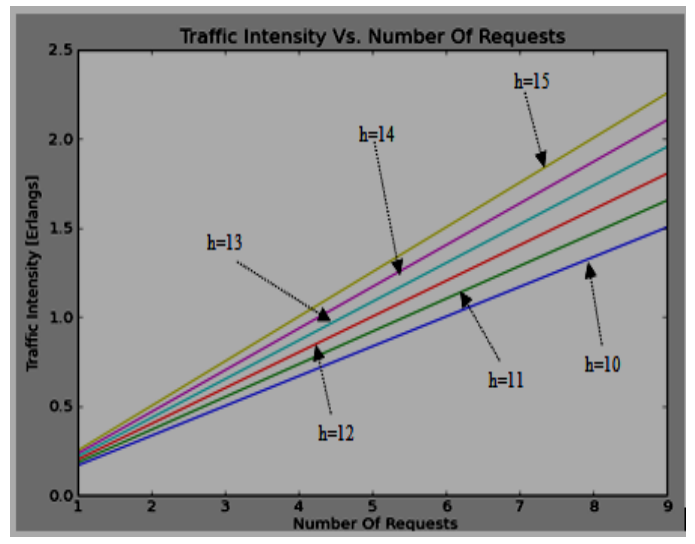

Fig.7. Variation of AC against Number of Request 
As Fig. 7 demonstrates, it was noted that increasing the holding time for the subscribers led to a corresponding increase in the traffic intensity. This is confirmed by the gradients when the call holding time was $11,12,13,14$ and 15 . Whereas call holding 10 had the lowest gradient, call holding time 15 had the steepest gradient. As such, the higher the call holding time per hour, the higher the value of the traffic intensity generated.

\section{Effect of BTS Channels Capacity on Blocking Probability}

The impact of the BTS channel capacity on the blocking probability was investigated by employing channel densities of 0 to 1400, and traffic density of 5 Erlangs, which was then increased up to a value of 10 Erlangs. The results obtained are shown in Fig. 8 that follows.

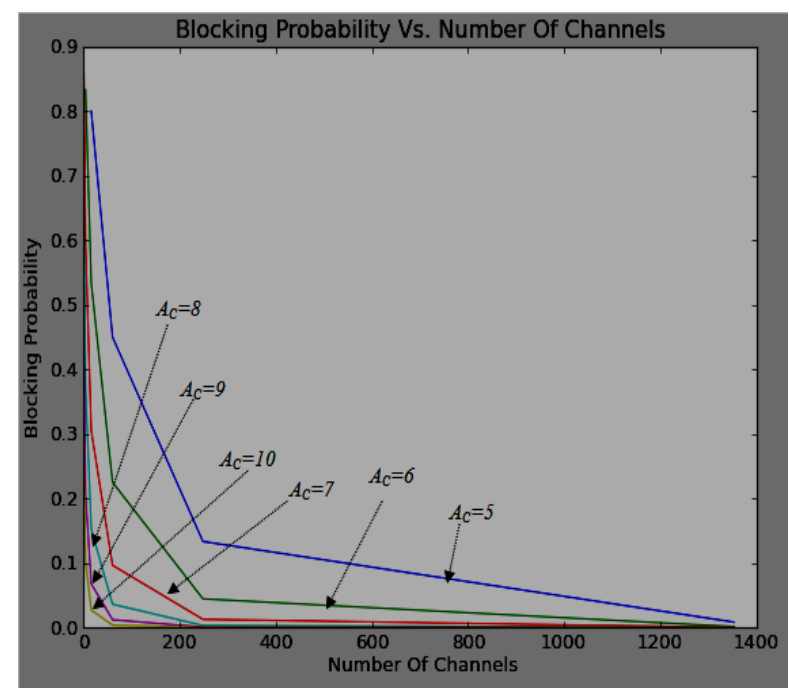

Fig.8. Effect of BTS Channels Capacity on Blocking Probability

As can be seen in Fig. 8, the steepest blocking was experienced when the network was loaded with 10 Erlangs of subscriber traffic while the lowest blocking probability gradient was observed when the network carried only 5 Erlangs. As the traffic density was slowly increased in factors of 1 Erlang, the network blocking probability gradient increased. In addition, it was observed that as the number of channels was increased from 0 to 1400 , the network blocking probability was reduced to almost zero at 1400 channel density. At channel level zero, the blocking probability was unity for all traffic densities.

\section{Impact of Offered Traffic On Blocking Probability}

In this section, the subscriber offered traffic densities were varied from zero to a value of 9 Erlangs and the impact of this was observed on the blocking probability. The number of subscribers $(n)$ were incremented from 7 to 10 and the effect of this increment was observed on the shape of the graph obtained. Fig. 9 that follows shows the results obtained.

As shown in Fig. 9, offered traffic had an influence on the network blocking probability. As the traffic was increased, the graph assumed a plateau approaching a blocking probability of 0.9 . Interesting to note was that for each value for the number of subscribers, the graphs assumed the same shape, rising sharply from blocking probability of zero, curving towards the plateau as the traffic offered is incremented. The subscriber density of 
$10(n=10)$ was observed to have the steepest ramp factor while a subscriber density for $7(n=7)$ had the least ramp factor.

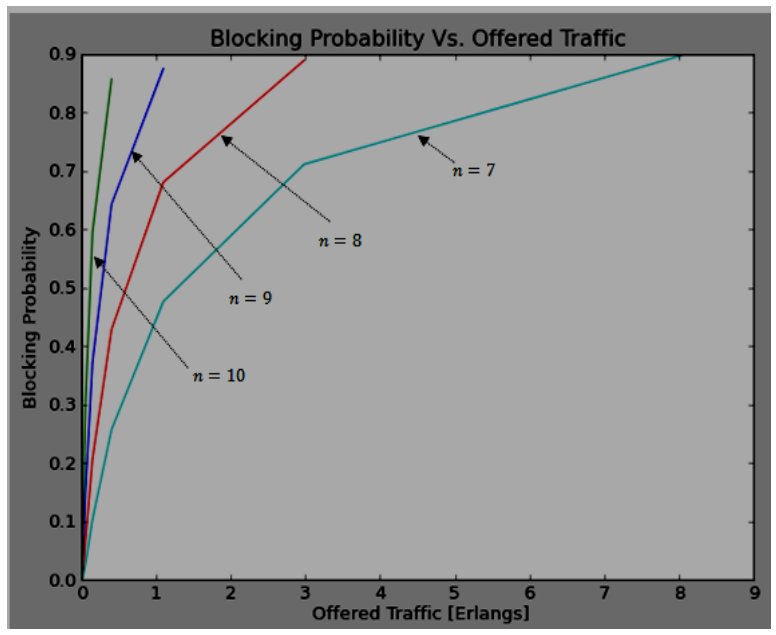

Fig.9. Impact of Offered Traffic on Blocking Probability

The interpretation is that if a large number of subscribers arrive at the same time in a region covered by a certain BTS, then they can easily cause a sharp rise in blocking probability of the BTS if they all initiate calls, or if they were already in the communication process and their calls experience a soft handover to this new cell. This is confirmed by comparing the behavior of the graphs for $n=10$ and $n=7$. For the former case, the ramp factor is very steep, nearly a straight line as it rises towards the highest blocking probability of 0.9 . However, for the latter case, the graph rises gently and at traffic load of 3 Erlangs, it appears to have reached a plateau and therefore rises more slowly towards the highest blocking probability of 0.8 . Consequently, when a small number of subscribers arrive at a given BTS, the impact of their new and handed over calls on the network blocking probability is low.

\section{E. Influence of Subscriber Distribution Density on Blocking Probability}

To establish the effect of subscriber distribution on the GSM network blocking probability, the number of subscribers was increased from zero to 10 . In addition, the traffic generated by these subscribers was varied from 0.5 Erlangs to 3.0 Erlangs and the shape of the graph was observed as shown in Fig. 10.

As Fig. 10 illustrates, the shapes of the blocking probability for all subscriber distribution under different traffic loads were fairly similar, maintain a blocking probability of zero and rising sharply when the number of subscribers hit a value of 9. At low subscriber distribution, the network blocking probability was nearly zero, only starting to show some blocking when their number approached 5. Considering traffic loads, when each subscriber was generating an average of 0.5 Erlangs, it took many of them to bring some blocking to the network. However, when each of them generated 3.0 Erlangs of traffic, their contribution to network blocking was significant, even as low as 6 of them. 


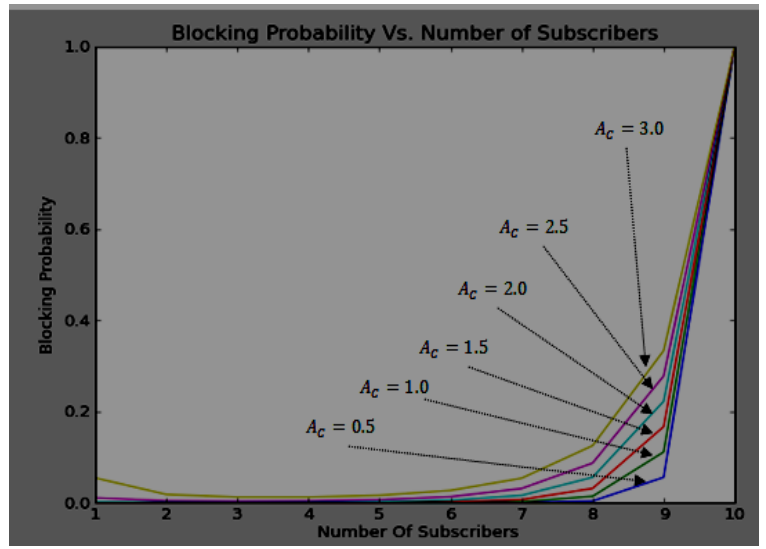

Fig.10. Effects of Subscriber Distribution on Blocking Probability

\section{F. Effects of Queued Traffic On Handover Blocking Probability}

In this scenario, a GSM system with prioritized handover scheme was considered. The queue length $\left(Q_{L}\right)$ was hypothesized to accommodate 60 Erlangs of traffic and the number of handover requests $\left(H_{R e q}\right)$ was varied from 10 to 25, being increased by 5 each time. The results obtained are shown in Fig. 11 below.

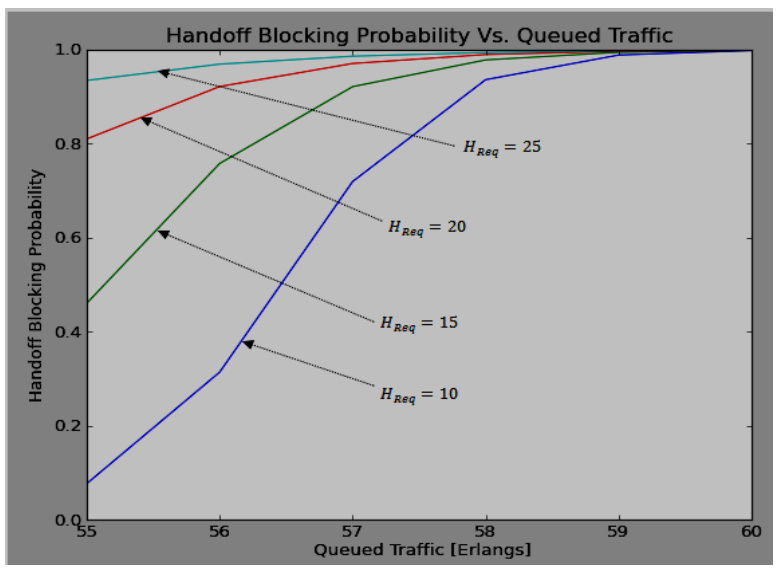

Fig.11. Effects of Queued Traffic on Handover Blocking Probability

Fig. 11 demonstrates that the amount of queued traffic indeed has an effect on handover request blocking probability. In addition, the number of handover requests also impacted on the handover blocking probability. It is interesting to note that all the graphs for various values of handover requests had similar shapes, converging slowly to the handover probability of unity (1) at queue length 60. Considering 10 handover requests $\left(H_{R e q}=10\right)$ and 25 handover requests $\left(H_{R e q}=25\right)$, it is noted that the handover blocking probability for the former is lower compared to the handover blocking probability for the latter. As such, the higher the handover request and the higher the number of queued traffic, the larger is the probability that new handover requests will be blocked and vice versa. 


\section{G. Impact of SINR on Orthogonal FDMA Blocking Probability}

To investigate how the SINR impacted on the orthogonal FDMA blocking probability, the SINR generated the GSM network was varied from 0 to 5 decibels $(\mathrm{dB})$ as the value of blocking probability was observed. The results obtained are shown in Fig. 12 below.

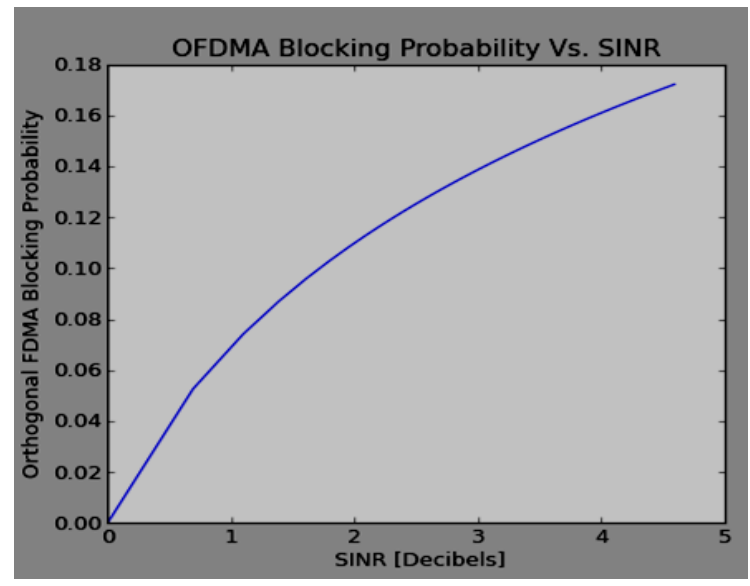

Fig.12. Impact of SINR on OFDMA Blocking Probability

As shown in Fig. 12, the value of the blocking probability is increased exponentially as the SINR is gradually increased from zero to a value of $5 \mathrm{~dB}$. The interpretation is that when noise signals arising from various factors such as cross talk in the GSM cellular network grow beyond a given limit, the subscriber signal quality can degrade to a point where this call is immaturely terminated. This is because GSM receiver have the minimum signal levels below which the call is either terminated or handed over to the next cell.

\section{H. Influence of Subscriber Call Redial Probability on Service Rates.}

Some subscribers are characterized by impatience, meaning that immediately their calls are immaturely terminated by the network, they usually initiate redials in order to continue with their communication. To investigate this phenomenon, the subscriber retry probability was varied between two extremes, which were 0.1 to 0.9 and the corresponding service rates were observed. Table 2 gives the results obtained.

Table 2. Variation of Service Rates with Retry Probability

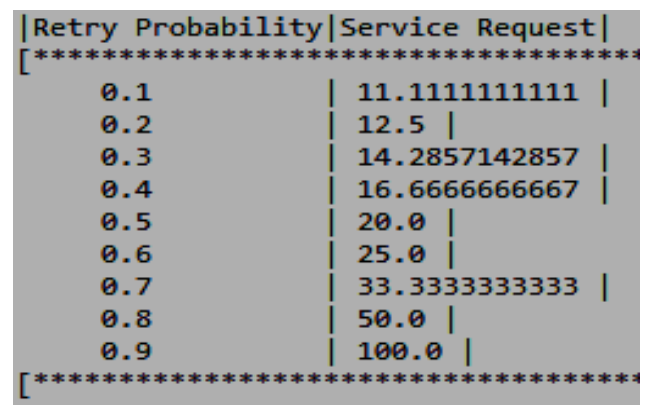


As Table 2 shows, different values for retry probability influenced the service requests generated by the subscribers. The nature of these effects was further probed under different subscriber arrival rates $(\tau)$. The value of the arrival rates was assumed to be a Poisson process which was increased in steps of two from a value of 10 to 16 arrivals per hour. Fig. 13 gives an illustration of the outcomes observed.

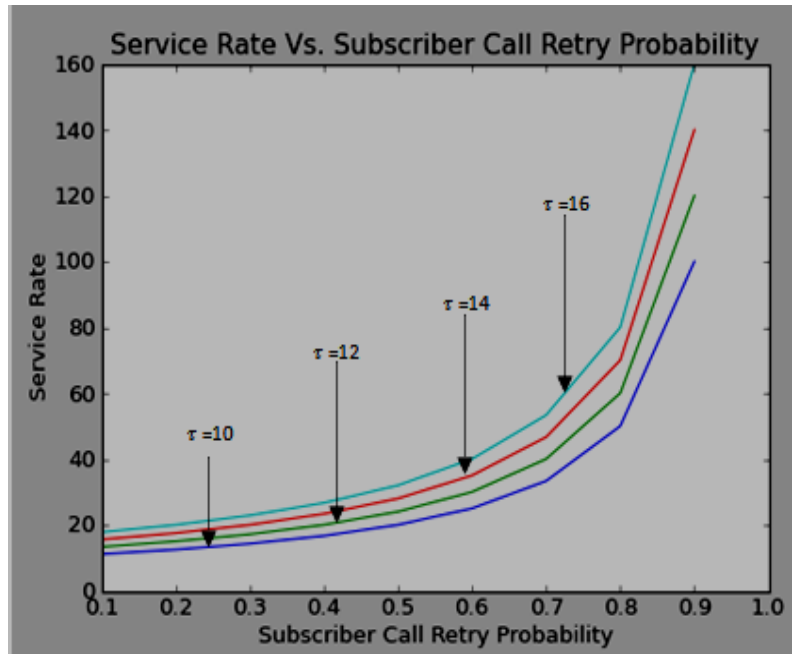

Fig.13. Effects of Subscriber Retry Probability on Service Rates

From Fig. 13, it can be observed that the subscriber retry probabilities for different subscriber arrival rates had the same shape, growing gradually at low call retry probabilities and then sporadically as the call retry probabilities approached the 0.8 level. Comparing the graphs for various subscriber arrival rates, then it was noted that with an arrival rate of 16 , the service rates were the greatest, hitting a maxima of 160 while for the arrival rate of 10 , the service rate was lowest, hitting a maximum rate of around 99 . The interpretation is that when a large group of subscribers arrive in a particular BTS and their services are terminated may be because of hard handover process, then if their call retry probability is high, the services rate shoots to high values, which may lead to call blocking for some of these redials and even new calls.

\section{Conclusions}

This paper sought to provide a probabilistic synthesis of GSM teletraffic blocking with Poissonian distribution arrival rates. A number of parameters which had previously been omitted in the commonly employed Erlang B and Erlang $\mathrm{C}$ have been synthesized and the corresponding blocking probabilities derived. Towards the end, numerical results emanating from the implementation of these blocking probabilities has been provided. It has been noted that with the increasing number of subscribers in a given region, then the Poissonian arrival approaches a normal distribution. The subscriber holding time has been established to affect traffic intensity whereby long call holding times led to increased traffic intensity. The effect of GSM channels availability on blocking probability has been investigated and has been observed to lead to high blocking probability when these channels are less. High offered traffic has been established to contribute to higher blocking probabilities while large subscriber distribution density was noted to contribute to high blocking probability. It was observed that high amounts of queued traffic and high handover request led to large handover blocking probabilities. In addition, it was noted that SINR contributed to an increase in blocking probability for orthogonal FDMA. On its part, subscriber call retry probability, when high, led to a sporadic increase in service request rates. 


\section{References}

[1] Osahenvemwen O \& Odiase F. (2016).Effective management of handover process in mobile communication network. Journal of Advances in Technology and Engineering Studies. Vol. 2, Issue 6, pp. 176-182.

[2] Kumar S., Kumar K., \& Kumar K. (2015). Mobility Based Call Admission Control and Resource Estimation in Mobile Multimedia Networks Using Artificial Neural Network, In 1st International Conference on Next Generation Computing Technologies. Pp. 852-857.

[3] Oliver M., Mohammad S., Ben O., \& Vinaye A. (2011). A Study Of Voice Traffic Blocking In A Model Cellular Network. International Journal of Wireless \& Mobile Networks. Vol. 3, No. 6, pp. 101-115.

[4] Anuj Kumar, Shilpi Srivastav, Alok Agarwal, Narendra Kumar (2012).Analysis of Blocking Probability in a GSM Based Cellular Network System. International Journal of Engineering Trends and Technology. Vol. 3, Issue5, pp. 601-604.

[5] Ambreen F., Pankaj K., \& Raj G. (2016). Reducing Handoff Blocking Probability in Wireless Cellular Networks using Auxiliary Stations and TDMA. International Research Journal of Engineering and Technology. Vol. 3 Issue 11, pp. 1249-1253.

[6] Sasibhushana R., Venkata K., \& Satya P. (2016). Teletraffic and Blocking Probability Estimation of OFDMA System. International Conference on Computational Modeling and Security. Vol. 85, pp. 696704.

[7] Biswajit B. (2013). A comparison study on selective traffic models with handoff management scheme for wireless mobile network infrastructure. International Journal of Information Technology and Computer Science. Vol.2, pp.66-72.

[8] Jatin K. (2016). Study and Analysis of Call dropping and Handover Problem in cellular system. International Journal of Advanced Research in Computer Engineering \& Technology. Vol. 5, Issue 6, pp. 1776-1777.

[9] Gangwar A. \& Singh V. (2014). Quality of Service Improvement, Handoff Prioritization and Channel Utilization for Cellular Network. International Journal of Engineering Research Applications. Vol. 4, Issue 10, pp. 46-49.

[10] Muhammad K., Abdul B., Manzoor K., Murad H.1,\& Mustajab A. (2016). Handover in GSM and WiMAX. International Journal of Advanced Research in Computer Engineering \& Technology. Vol. 5, Issue 6, pp. 2034-2041.

[11] Sameera W., Senarath S., Dhishan D., Weerarathne A., \& Peiris G. (2016). Efficient Handoff for Mobility and Security for GSM. Imperial Journal of Interdisciplinary Research. Vol-2, Issue-5, pp. 11821189.

[12] Adeyinka A., Samuel N., \& Emmanuel R. (2016). Performance Comparison of Dynamic Guard Channel Assignment with Buffered Prioritized Scheme for Mobile WiMAX Network. Proceedings of the World Congress on Engineering. Vol. I, pp. 1-5.

[13] Galadima A., Dajab D., \& Bajoga G. (2014).The Analysis Of Inter Cell Handover Dynamics in A GSM Network. International Journal of Innovative Research in Science, Engineering and Technology. Vol. 3, Issue 6, pp. 13444-13451.

[14] Arul F., \& Vidhya V.(2017). Handoff Management in Priority Based Feedback Cellular Networks with Catastrophes and Impatient Customers. International Journal of Innovative Research in Science, Engineering and Technology. Vol. 6, Issue 1, pp. 1021-1025.

[15] Na L. (2016). Recent Advances in Accumulating Priority Queues. Doctorate Thesis, University of Western Ontario: Electronic Thesis and Dissertation Repository. Pp.1-169. 
[16] Sharif A., Stanford D., Taylor P., \& Ziedins I. (2014). A multi-class multi-server accumulating priority queue with application to health care. Operations Research for Health Care. Vol. 3, Issue 2, pp.73-79.

[17] Adeyinka A., Victor O., Emmanuel R., Adebiyi A.,\& Samuel N.(2016). Performance Evaluation of Dynamic Guard Channels Assignment with Queue and Prioritized Schemes. International Conference on Computational Science and Computational Intelligence. Pp. 1055-1063.

[18] Malathy E., \& Vijayalakshmi M. (2016). Vertical Handover Facilitation Through Queuing Model In Heterogeneous Wireless Network. International Journal of Advanced Engineering Technology. Vol.8, Issue I, pp. 346-348.

[19] Luae A., \& Sareh T. (2017). Minimizing Blocking Probability in Elastic Optical Networks by Varying the Bandwidth Granularity Based on Optical Path Fragmentation. Photonics. Vol 4, Issue 20, pp. 1-14.

[20] Nadine K., Mirna A., \& Ali H. (2016). Identifying the Effective Parameters for Vertical Handover in Cellular Networks Using Data Mining Techniques. The 7th International Conference on Emerging Ubiquitous Systems and Pervasive Networks. Vol. 98 , pp. 91 - 99.

[21] Sanjeev K., Krishan K., \& Anand K. (2016). Dynamic Channel Allocation in Mobile Multimedia Networks Using Error Back Propagation and Hopfield Neural Network (EBP-HOP). Twelfth International Multi-Conference on Information Processing. Vol. 89, pp.107 - 116.

\section{Authors' Profiles}

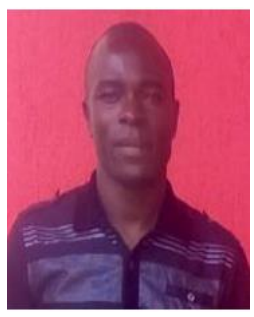

Vincent O. Nyangaresi, Bsc (TIT), Msc (IT Sec. \& Audit) is a researcher in areas of data communication and computer networks, network design and administration, distributed systems and information systems security. He is currently pursuing his $\mathrm{PhD}$ in Information Security and Audit. He has published numerous research articles covering areas such as communication systems, secure network communications, information systems acceptance modeling, TCP architecture and design, radio wave propagation, virtualization and cloud computing, among others. In addition, he lecturers in the fields of computer networks, digital forensics, software engineering, digital electronics and applied computer science.

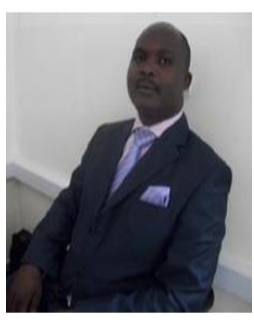

Dr. Silvance O. Abeka is currently the Dean, School Of Informatics And Innovative Systems, Jaramogi Oginga Odinga University of Science And Technology. He holds a masters degree in Business Administration (Information Techn- ology) and a PhD in Management Information Science (MIS), from Kampala International University, Dar es Salaam Collage. His interests include Management Information Systems, Principles of Statistics and E- Commerce. He is also a lecturer in the school of Computer Studies.

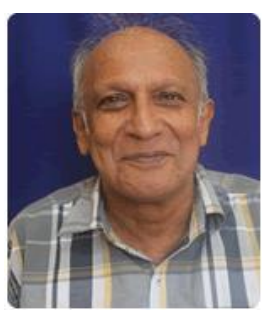

Prof. Anthony Rodrigues, (University of Manchester, institute of science \& technology, England). He has lectured in a number of universities and is currently a director, directorate of information and communications technology of Jaramogi Oginga Odinga University of science and technology. His research interests include scientific computation, approximation theory, modeling, informatics policy among others. 
How to cite this paper: Vincent Omollo Nyangaresi, Silvance Abeka, Anthony Rodrigues, " Multivariate Probabilistic Synthesis of Cellular Networks Teletraffic Blocking with Poissonian Distribution Arrival Rates", International Journal of Wireless and Microwave Technologies(IJWMT), Vol.8, No.4, pp. 14-39, 2018.DOI: 10.5815/ijwmt.2018.04.02 